ARTICLE

\title{
Structural basis of suppression of host translation termination by Moloney Murine Leukemia Virus
}

\author{
Xuhua Tang ${ }^{1}$, Yiping Zhu ${ }^{2,3}$, Stacey L. Baker ${ }^{4}$, Matthew W. Bowler ${ }^{5,6}$, Benjamin Jieming Chen ${ }^{1}$, Chen Chen ${ }^{1}$,
} J. Robert Hogg ${ }^{4}$, Stephen P. Goff ${ }^{2,3}$ \& Haiwei Song ${ }^{1,7,8}$

Retroviral reverse transcriptase (RT) of Moloney murine leukemia virus (MoMLV) is expressed in the form of a large Gag-Pol precursor protein by suppression of translational termination in which the maximal efficiency of stop codon read-through depends on the interaction between MoMLV RT and peptidyl release factor 1 (eRF1). Here, we report the crystal structure of MoMLV RT in complex with eRF1. The MoMLV RT interacts with the C-terminal domain of eRF1 via its RNase $\mathrm{H}$ domain to sterically occlude the binding of peptidyl release factor 3 (eRF3) to eRF1. Promotion of read-through by MoMLV RNase $\mathrm{H}$ prevents nonsense-mediated mRNA decay (NMD) of mRNAs. Comparison of our structure with that of HIV RT explains why HIV RT cannot interact with eRF1. Our results provide a mechanistic view of how MoMLV manipulates the host translation termination machinery for the synthesis of its own proteins.

\footnotetext{
${ }^{1}$ Institute of Molecular and Cell Biology, 61 Biopolis Drive, Proteos, Singapore 138673, Singapore. ${ }^{2}$ Department of Biochemistry and Molecular Biophysics, Columbia University, HHSC 1310C, 701 West 168th Street, New York, New York 10032, USA. ${ }^{3}$ Howard Hughes Medical Institute, Columbia University, HHSC 1310C, 701 West 168th Street, New York, NY 10032, USA. ${ }^{4}$ Biochemistry and Biophysics Center, National Heart, Lung, and Blood Institute, National Institutes of Health, 50 South Drive, Bethesda, Maryland 20892, USA. ${ }^{5}$ European Molecular Biology Laboratory, Grenoble Outstation, 71 Avenue des Martyrs, CS 90181, Grenoble F-38042, France. ${ }^{6}$ Unit of Virus Host-Cell Interactions, University Grenoble Alpes-EMBL-CNRS, 71 Avenue des Martyrs, CS 90181 , Grenoble F-38042, France. ${ }^{7}$ Life Sciences Institute, Zhejiang University, 388 Yuhangtang Road, Hangzhou 310058, China. ${ }^{8}$ Department of Biochemistry, National University of Singapore, 14 Science Drive, Singapore 117543, Singapore. Correspondence and requests for materials should be addressed to J.R.H. (email: j.hogg@nih.gov) or to S.P.G. (email: spg1@cumc.columbia.edu) or to H.S. (email: haiwei@imcb.a-star.edu.sg).
} 
$\mathrm{D}$ ue to limited genome encoding capacity, viruses are reliant on the host translation system for synthesis of their viral proteins. Various viruses have been found to manipulate almost every step of the host translation process, mainly through targeting cellular translation factors ${ }^{1}$. Most retroviruses utilize translational recoding of a viral messenger RNA (mRNA) stop codon to express the gag gene both as an independent polyprotein (Gag) and as a fusion (Gag-Pol) with the polyprotein encoded by the pol gene. The ratio of Gag to Gag-Pol is delicately balanced during virus assembly and is critical for infection ${ }^{2,3}$. Therefore, retroviruses have developed at least two different strategies to switch between Gag and Gag-Pol expression, both regulated by viral pseudoknots or hairpin RNA structures $^{4-8}$. In HIV, the gag and pol genes are in different reading frames and Gag-Pol protein production requires a -1 ribosomal frameshift at the end of the gag gene. However, in Moloney murine leukemia virus (MoMLV), these two genes are in the same reading frame, and the suppression of the gag UAG termination codon permits read-through to the pol gene 9 . The essential replication enzyme reverse transcriptase (RT) is expressed as part of a Gag-Pol fusion protein, accounting for $5 \%$ of unspliced retroviral RNA translation. Biochemical and structural studies showed that the MoMLV RT displays a monomeric architecture containing an $\mathrm{N}$-terminal polymerase domain and a C-terminal RNase $\mathrm{H}$ domain (Fig. 1a) ${ }^{10}$. In contrast, the HIV RT is a heterodimer consisting of p66 and p51 subunits ${ }^{11}$, the latter of which is derived by proteolysis of p66 and lacks the RNase $\mathrm{H}$ domain.

In eukaryotes, stop codon recognition is initiated by the binding of the essential termination factor eRF1 in complex with a guanosine triphosphate (GTP)-bound eRF3 to the ribosome. The subsequent dissociation of eRF3 after GTP hydrolysis and recruitment of ATP binding cassette E1 (ABCE1) to eRF1 induce peptide release and ribosomal subunit dissociation ${ }^{12}$. Comprehensive structural studies of eRF1 and its complexes with other termination factors have enabled an understanding of how eRF1 coordinates the termination events via its three distinct domains ${ }^{13-18}$, namely the N-terminal domain (eRF1-N), M-domain (eRF1-M) and C-terminal domain (eRF1-C) (Fig. 1a). eRF1-N (aa 1-142) contains a conserved Asn-Ile-Lys-Ser (NIKS) motif and has an essential role in stop codon recognition ${ }^{13}$. eRF1-M (aa 143-276) mimics the transfer RNA (tRNA) acceptor stem and harbours a universal GGQ tip to stretch toward the CCA end of the peptidyl tRNA for triggering peptide release ${ }^{13,17}$. eRF1-C (aa 277437) functions to recruit other termination factors such as eRF3 when forming the pre-termination complex ${ }^{13,14,16}$.

Previously, we showed that MoMLV utilized its RT to interact with host eRF1, thereby promoting stop codon read-through to make Gag-Pol ${ }^{19}$. However, the molecular events underlying this mechanism remain undefined. In this study, we determine the crystal structure of MoMLV RT in complex with the full-length mouse eRF1. Our structure shows that the MoMLV $\mathrm{RT}$ interacts with the C-terminal domain of eRF1 via its RNase $\mathrm{H}$ domain. Structure-guided functional assays suggest that MoMLV RT suppresses translation termination by outcompeting eRF3 for binding to eRF1. We also show that MoMLV RNase $\mathrm{H}$ promotes stop codon read-through, which in turn prevents nonsense-mediated mRNA decay (NMD). Altogether, these results reveal the structural basis of host translation termination suppression by MoMLV.

\section{Results}

Structural determination. We solved the crystal structure of MoMLV RT (aa 24-671) in complex with the full-length mouse eRF1 at a resolution of $4.0 \AA$ (for simplicity designated as
MoMLV RT/eRF1) (Fig. 1b). MoMLV RT contains an N-terminal polymerase domain and C-terminal RNase $\mathrm{H}$ domain connected by a flexible linker, which is not visible in the electron density map and is assumed to be disordered. Structural comparison of the RT polymerase domain in our structure with that in a MoMLV RT/DNA complex ${ }^{20}$ and a Xenotropic murine leukemia virus-related virus (XMRV) RT/RNA/DNA ternary complex ${ }^{10}$, reveals that the binding of eRF1 did not cause a significant conformational change in MoMLV RT's polymerase domain (Supplementary Fig. 1a). The catalytic residues (D524, E562, D583 and D653) of RNase H are distant from the bound eRF1 (Supplementary Fig. 1b). The RNase H activity assays further confirmed that eRF1 binding does not affect the RNase $\mathrm{H}$ activity (Supplementary Fig. 1c, upper panel). These findings are consistent with our previous data that the MoMLV RT-eRF1 interaction does not affect RT's activities ${ }^{19}$.

The MoMLV RT-eRF1 interaction is mediated via the contacts of the RNase $\mathrm{H}$ domain of MoMLV RT with the C-terminal domain of eRF1. To verify this interaction, we also determined the crystal structure of the isolated RNase $\mathrm{H}$ domain of MoMLV $\mathrm{RT}$ in complex with eRF1-C at a resolution of $2.8 \AA$ (Fig. 1c).
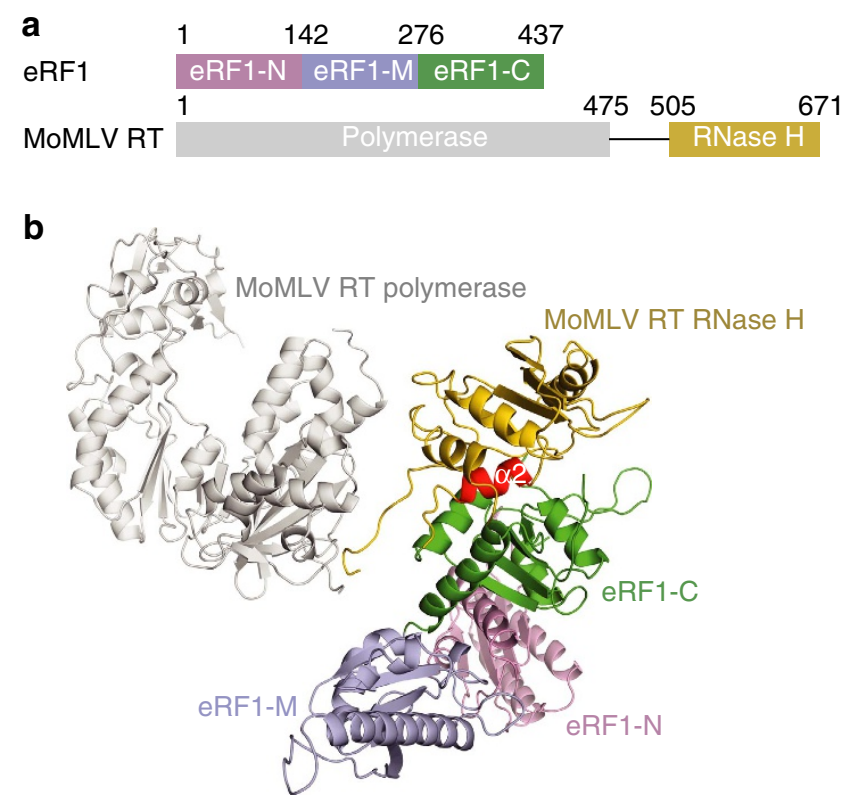

C

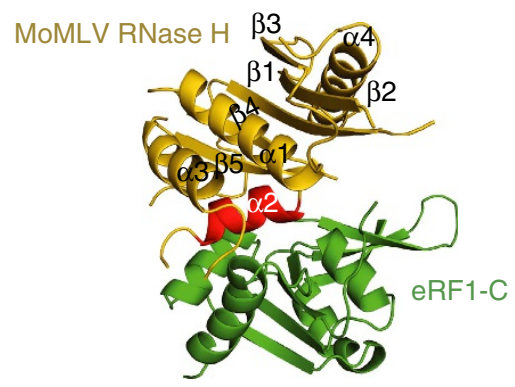

Figure 1 | Structure of the MoMLV RT/eRF1 complex. (a) Schematic representation of the domain organization of eRF1 and MoMLV RT. Domains $N, M$, and C of eRF1 are coloured in pink, lightblue and green, respectively. MoMLV RT polymerase domain is coloured in grey and RNase $\mathrm{H}$ domain in yellow. (b) A ribbon diagram of the MoMLV RT/eRF1 complex. The colouring scheme is as in $\mathbf{a}$. The helix $\alpha 2$ of RNase $\mathrm{H}$ domain is highlighted in red. (c) The RNase H/eRF1-C complex structure. The secondary structure elements of RNase $\mathrm{H}$ domain are labelled. 
As the RNase H/eRF1-C interface is identical with that observed in the MoMLV RT/eRF1 complex, subsequent analysis concerning the RT-eRF1 interaction only refers to the RNase $\mathrm{H} / \mathrm{eRF} 1-\mathrm{C}$ structure.

Interaction of MoMLV RT with eRF1. The interaction of the RNase $\mathrm{H}$ domain of MoMLV RT with eRF1-C buries a total solvent accessible surface area of $1173 \AA^{2}$. The RNase H domain mainly uses its helix $\alpha 2$ to interact with eRF1-C through predominantly hydrophobic contacts (Fig. 2a). Residues Phe588, Ala589, Ile593 and the methylene group of Arg585 in helix $\alpha 2$ of the RNase $\mathrm{H}$ domain form a hydrophobic patch that interacts with the hydrophobic residues of eRF1-C, including Phe291 and Ile294 in helix $\alpha 8$, Tyr301 and Phe303 in the loop region connecting $\alpha 8-\alpha 9$ and Phe406 in loop $\alpha 11-\beta 11$ (numbering as previously described ${ }^{16}$ ). In addition to these predominant hydrophobic interactions, Arg585 of the RNase $\mathrm{H}$ domain forms a salt bridge with Asp307 of eRF1-C, Gln559 of RNase H is hydrogen bonded to Asp297 of eRF1-C, and Asp511 of RNase $\mathrm{H}$ forms hydrogen bonds with the carbonyl groups of Lys404 and Gly407 in helix $\alpha 11$ of eRF1-C.

To examine the role of the RNase H/eRF1-C interface, we mutated several residues and examined their effects on binding by glutathione S-transferase (GST) pull-down assays (Fig. 2b). Mutation of Ile294, Tyr301 or Phe406 of eRF1-C to alanine disrupted its interaction with RNase H. Likewise, single mutations in the isolated RNase H domain (R585A, F588A and A589K) of MoMLV RT abolished its binding to eRF1-C (Fig. 2b). Residues Ile294, Tyr301, Phe406 of eRF1-C and Phe588 of RNase H are the key components of the hydrophobic RNase H/eRF1-C interface. Single Ala mutations of these residues disrupted the RNase H/eRF1-C interaction. Mutation of Arg585 to Ala would abolish its salt bridge interaction with Asp307, while substitution of Ala589 by a lysine would place a charged residue inside the hydrophobic interface, thereby destabilizing the RNase H/eRF1-C
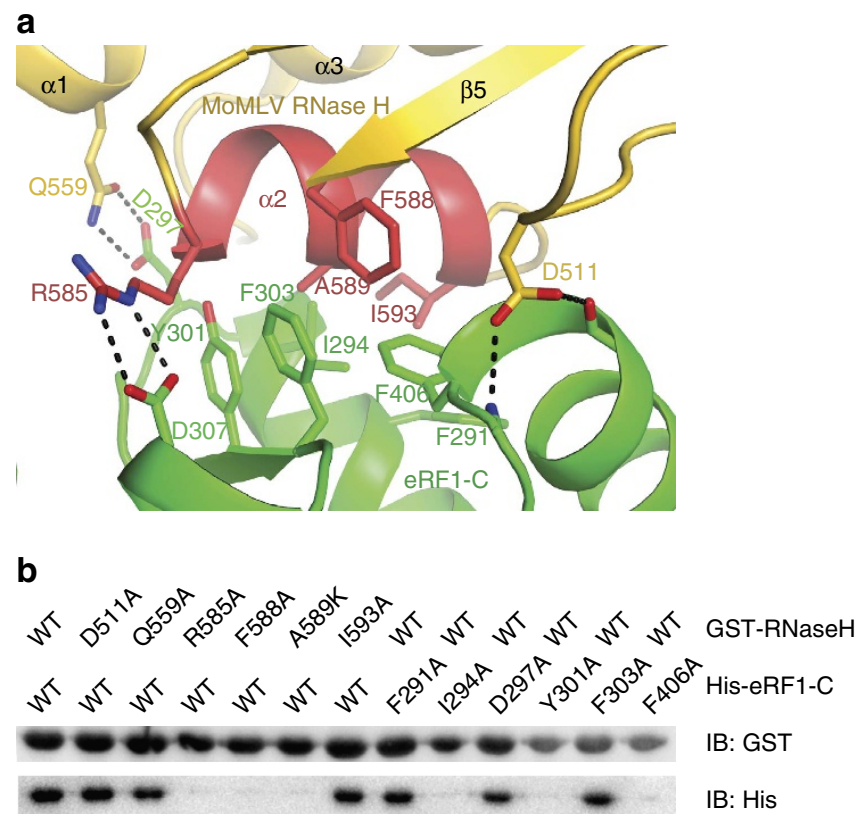

Figure 2 | Interaction of MoMLV RT with eRF1. (a) Interface between the RNase $\mathrm{H}$ domain of MoMLV RT and eRF1-C. Residues involved in the interaction are shown as sticks and labelled. (b) GST pull-down assay. GST tagged WT MoMLV RNase $\mathrm{H}$ and its mutants on beads were used to bind His-tagged eRF1-C and its variants. interaction. In contrast, single Ala substitutions of Asp511, Gln559 and Ile593 of RNase H and Phe291, Asp297 and Phe303 of eRF1 had no effect on the RNase H/eRF1-C interaction. Residues Asp511, Ile593 and Qln559 of RNase H, and Phe291 and Asp297 of eRF1 are located at the edge of the main interface and would not be predicted to contribute significantly to the RNase H/eRF1-C interaction, and therefore it is not surprising that mutations of these residues had no effect on the interaction. Phe303 of eRF1 is situated in the middle of the interface and appears to be a key hydrophobic residue. One plausible explanation for the F303A mutant of eRF1 lacking the RNase $\mathrm{H}$ binding defect is that the side chain of Arg585 could rotate and place its methylene group in a position to compensate for the loss of the phenol group of F303A mutant.

We further examined the binding thermodynamics of wild-type (WT) RNase $\mathrm{H}$ and its variants to eRF1 by isothermal titration calorimetry (ITC). WT RNase H binds to eRF1 with an equilibrium dissociation constant $\left(K_{\mathrm{d}}\right)$ of $\sim 4.18 \mu \mathrm{M}$. The RNase $\mathrm{H}$ mutant F588A showed $\sim 10$-fold reduced binding to eRF1, whereas the binding of R585A or A589K to eRF1 was abrogated (Supplementary Fig. $2 \mathrm{a}-\mathrm{d}$ ). These data indicate that helix $\alpha 2$ of the RNase $\mathrm{H}$ domain in MoMLV RT harbouring these residues is critical for the RT-eRF1 interaction.

We note that earlier mutational studies found that mutation G525E led to a strong disruption of RT-eRF1 binding as assessed in a yeast two-hybrid assay ${ }^{19}$. Gly525 is far from the RT-eRF1 interface and is located in the interior of the RNase $\mathrm{H}$ domain. Its substitution by a glutamate acid places a negatively charged residue with a long side chain inside the hydrophobic core of the RNase $\mathrm{H}$ domain, therefore destabilizing the fold of RNase $\mathrm{H}$. Thus, we suggest this mutation acts indirectly.

The interaction of polymerase domain of RT with eRF1 by GST pull-down assay was only detectable in low salt condition in the previous study ${ }^{19}$. Thus, the crystallization buffer conditions in this study may prevent the polymerase domain of RT from interacting with eRF1. Intriguingly, we observe an interface between the RT polymerase domain and eRF1 within the crystal lattice in our MoMLV RT/eRF1 structure, but only between symmetry-related molecules. We postulate that this observation may only be due to the crystal packing, because the electron density of this interface is not well defined, indicating a certain degree of disorder in this area.

The RT-eRF1 interaction enhances read-through. To determine the effect of the MoMLV RT-eRF1 interaction on translational read-through, we introduced the RT mutations into the MoMLV provirus and examined the expression levels of the Gag precursor protein and capsid protein (CA) in virion lysates. The readthrough efficiency is evaluated here by examination of the CA/ Gag ratio, as the processing of Gag precursor protein to CA protein is mediated by PR protease, which depends on readthrough for its expression. Compared with the WT virions, those with mutations affecting eRF1 binding (R585A, F588A or A589K) showed dramatically increased ratios of Gag precursor to mature CA protein, indicating that a large fraction of the Gag precursor proteins remained unprocessed (Fig. 3a). Although we cannot rule out other mechanisms for the effects of the RT mutations, the results are consistent with a reduced expression of read-throughdependent PR protease present in the Gag-Pol precursor. The viruses were then used to infect Rat 2 cells to detect their ability to spread. Although WT viruses rapidly spread in the cultures, all mutants were replication defective (Fig. 3b). This finding is consistent with the notion that the interaction with eRF1 is important for replication, as these mutations have little or no 
a

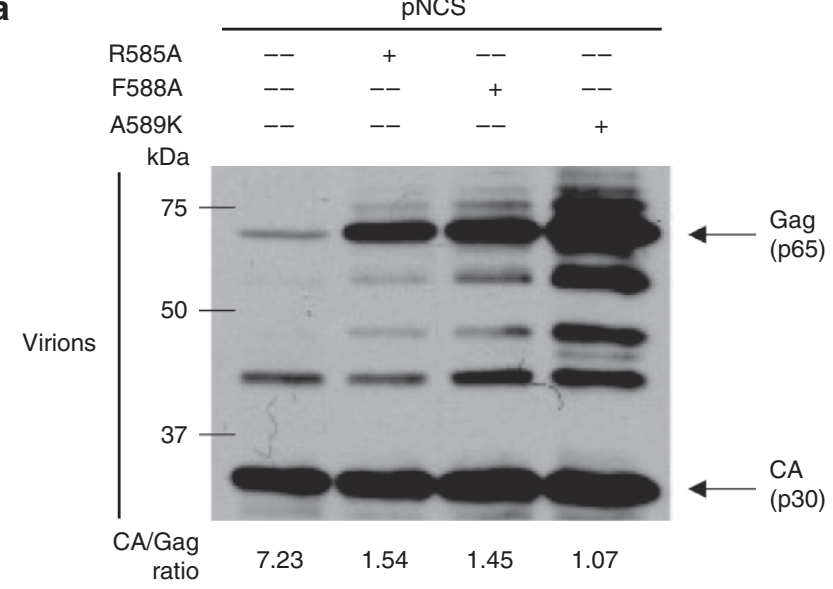

b

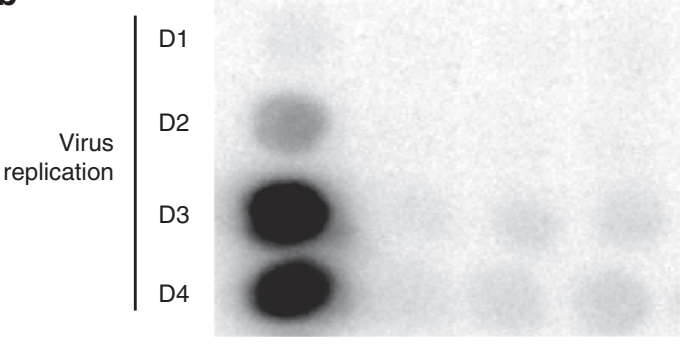

C

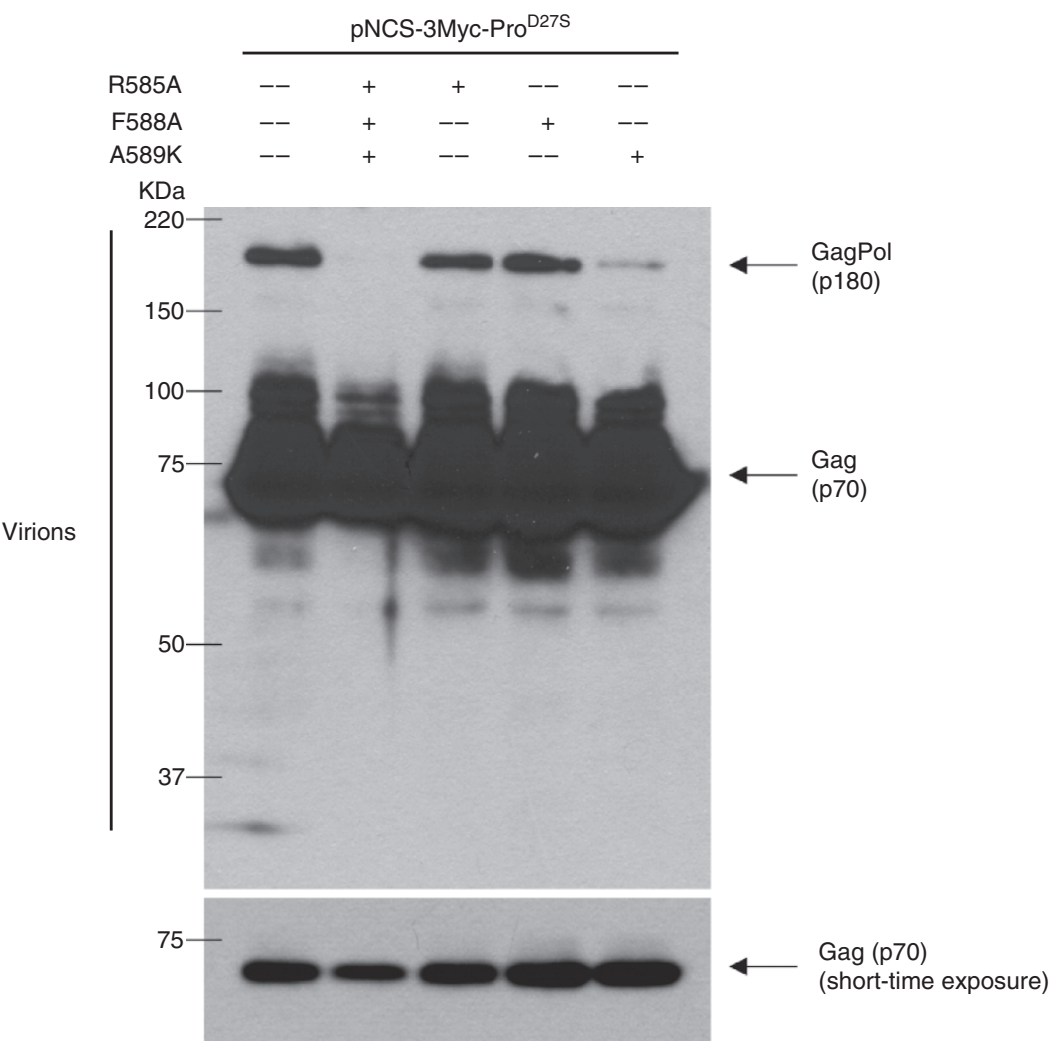

Figure 3 | Non-interacting RT mutants showing reduced read-through are replication defective. (a) The provirus carrying mutants that are unable to bind eRF1 show reduced read-through efficiency. The proviral DNA were used to transform $293 \mathrm{~T}$ cells and the purified virions were analyzed by western blot. The virion produced by WT DNA contained high levels of capsid (CA, 30 kDa), while mutants showed poor Gag ( $\sim 65 \mathrm{KDa}$ ) cleavage as a consequence of low read-through. (b) Non-interacting RT mutants are replication defective. Release of spreading virus was detected on the indicated day

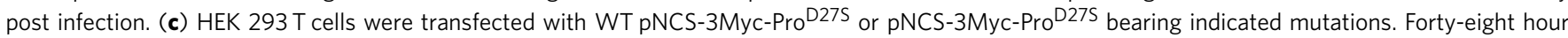
after transfection, the viruses in the supernatant were collected and purified by ultra-centrifuge through the $25 \%$ sucrose cushion. The virus pellets were re-suspended in protein loading buffer, resolved in SDS-PAGE, and visualized by western blot using c-Myc antibody (9E10; Sc-40, Santa Cruz; 1:1000 dilution). Noted that the Gag protein has a molecular mass of $\sim 70 \mathrm{kDa}$ as carrying the $3 \mathrm{Myc}$ tag. 
effect on their RNase $\mathrm{H}$ activities (Supplementary Fig. 1c, lower panel). To further examine the role of the RT-eRF1 interaction in read-through, selected mutations (R585A, F588A or A589K) were introduced into an MoMLV provirus in which the protease catalytic site was mutated (Pro ${ }^{\mathrm{D} 27 \mathrm{~S}}$ ). In this context, the uncleaved Gag-Pol precursor with WT RT was readily detected in virion particles, while the Gag-Pol protein with triple mutations (R585A/F588A/A589K) was undetectable. The A589K mutation significantly reduced Gag-Pol levels, while the R585A or F588A mutations had no effect on Gag-Pol levels (Fig. 3c). We conclude that A589 is the most important amino acid for RT-mediated enhancement of read-through. These observations support the hypothesis that the read-through-enhancing interaction of RT with $\mathrm{eRF} 1$ is required for viral replication.

MoMLV RT outcompetes eRF3 for binding to eRF1. eRF1 and eRF3 interact predominantly via their respective C-terminal domains (eRF1-C and domain 3 of eRF3) and bind to the ribosomal pre-termination complex as a stable eRF1/eRF3 complex for stop codon decoding ${ }^{15,16}$. Structural superposition of MoMLV RT/eRF1 and eRF1/eRF3 complexes showed that eRF1 interacts with both MoMLV RT and eRF3 through overlapping surface regions (Fig. 4a), suggesting that MoMLV RT and eRF3 are mutually exclusive for binding to eRF1. In support of this structural observation, WT RNase $\mathrm{H}$ but not mutants defective in eRF1 binding efficiently outcompetes eRF3 for binding eRF1 with an apparent $K_{\mathrm{d}}$ of $\sim 5.6 \mu \mathrm{M}$, despite eRF3 exhibiting at least 10 times higher affinity for eRF1 than RNase H (Fig. 4b,c; Supplementary Fig. 2e-g). These results suggest that MoMLV RT likely suppresses translation termination by outcompeting eRF3 for binding to eRF1.

Promotion of read-through by $\mathrm{RNase} \mathbf{H}$ prevents NMD. We introduced MoMLV or HIV RNase $\mathrm{H}$ immediately downstream of the MoMLV pseudoknot (MLVPK) in dual-fluorescent-protein reporters of translational read-through (Fig. 5a). These reporters mimic the natural context of RNase $\mathrm{H}$ expression, and could either allow activity in trans (on different mRNAs following termination and release) or in cis (at the upstream termination codon on the same mRNA). In the latter scenario, termination suppression activity may be enhanced by tethering of newly synthesized RNAse $\mathrm{H}$ via the nascent polypeptide. This positioning of the MoMLV RNase $\mathrm{H}$ domain led to $\sim$ twofold more read-through than control constructs containing mCherry alone or the HIV RNase $\mathrm{H}$ and mCherry downstream of the MoMLV pseudoknot. Stimulation of read-through by MoMLV RNase $\mathrm{H}$ was evident when using both the WT UAG stop codon and a UAA stop codon, which reduces read-through to $\sim 2 \%$ in controls. When moved to a position $3^{\prime}$ of the mCherry open reading frame (ORF), the MoMLV RNase $\mathrm{H}$ failed to stimulate read-through. Because polypeptides containing C-terminal RNase $\mathrm{H}$ would undergo termination and release immediately after completion of RNase $\mathrm{H}$ synthesis, this finding suggests that nascent polypeptide-mediated tethering of MoMLV RNase $\mathrm{H}$ to the translating mRNA is important for optimal function (Fig. 5b).

Termination at the MoMLV gag stop codon results in an mRNA with an effective $3^{\prime}$ UTR length of $\sim 6,000 \mathrm{nt}$, a feature expected to make the transcript susceptible to the conserved NMD pathway ${ }^{21}$ in the absence of a protective mechanism. As previous observations show that induction of read-through by the MoMLV pseudoknot can potently inhibit NMD of reporter transcripts ${ }^{22}$, we investigated the ability of MoMLV $\mathrm{RNase} \mathrm{H}$ to augment this activity in pulse-chase decay assays of tetracycline-regulated dual-fluorescent-protein reporters. Because degradation by NMD is strongly $3^{\prime} \mathrm{UTR}$ length-dependent, we used either HIV RNase $\mathrm{H}$ or NanoLuc luciferase (Nluc) to equalize $3^{\prime}$ UTR length among the constructs used. Read-through stimulated by the WT MoMLV pseudoknot (UAG) was sufficient to fully stabilize mRNAs with the extended mCherry-Nluc $3^{\prime}$ UTR, likely due to efficient displacement of the NMD key effector Upf1 from the $3^{\prime}$ UTR by elongating ribosomes ${ }^{22}$ (half-life calculated from best-fit line $=519 \mathrm{~min}, 95 \%$ confidence of interval $(\mathrm{CI})=423-670 \mathrm{~min})$. Therefore, to test a potential role for the RNase $\mathrm{H}$ domain in contributing to NMD inhibition, we used the impaired UAA MoMLV pseudoknot variant, which promoted partial rescue of mRNAs containing Nluc or HIV RNase H 3'UTR extensions (Nluc: best-fit $=286 \mathrm{~min}$, 95\% CI = 256-322 min; HIV RNase H: best-fit $=296 \mathrm{~min}, 95 \%$ $\mathrm{CI}=251-360 \mathrm{~min})$. Consistent with the read-through data above, the presence of the MoMLV RNase $\mathrm{H}$ dramatically increased RNA stability, resulting in a half-life of $\sim 530 \mathrm{~min}$ (95\% $\mathrm{CI}=457-653 \mathrm{~min}$ ). This half-life is similar to that exhibited by mRNAs containing the UAG pseudoknot and Nluc, consistent with a model in which promotion of read-through by MoMLV RNase $\mathrm{H}$ in turn prevents NMD (Fig. 5c).

\section{Discussion}

Translation termination and ribosome recycling are two critical processes in protein biosynthesis. During translation termination, a stop codon in the ribosomal A site is decoded by eRF1 delivered by eRF3 in the form of an eRF1/eRF3/GTP ternary complex. After GTP is hydrolyzed, eRF3 dissociates from the ribosome, and ABCE1 binds to the eRF1-bound ribosome to stimulate peptide release and subsequent ribosomal subunit dissociation ${ }^{23,24}$. Structural analysis showed that eRF1 uses its C-terminal domain to interact with both eRF3 and ABCE1 (refs 13,16). Our structural and functional studies of the MoMLV RT/eRF1 complex showed that MoMLV RT likely suppresses stop codon read-through via outcompeting eRF3 binding to eRF1. Since the same surface of eRF1 is used for binding both eRF3 and ABCE1, MoMLV RT would be predicted to compete with ABCE1 for binding to eRF1, therefore inhibiting peptide release and possibly ribosomal subunit dissociation as well. In this scenario, the interval between eRF3 GTP hydrolysis/dissociation and ABCE1 binding would give MoMLV RT a window of time to interact with and inhibit eRF1.

Retroviruses appear to have evolved multiple mechanisms to protect their mRNAs from NMD. Recent data show that a cis-acting RNA stability element immediately downstream of gag in unspliced Rous sarcoma virus (RSV) RNA recruits polypyrimidine tract binding protein 1 to protect its long $3^{\prime} \mathrm{UTR}$ from $\mathrm{NMD}^{25}$. In deltaretrovirus human $\mathrm{T}$-lymphotropic virus type 1, it was observed that the viral protein Tax inhibits mRNA degradation via interacting with INT6 and preventing the association of INT6 with Upf1 (ref. 26). A possible alternative retroviral mechanism for NMD escape was suggested by findings that insertion of the read-through-promoting MLVPK sequence into NMD reporter transcripts antagonizes Upf1 recruitment and subsequent mRNA decay ${ }^{22}$. In addition to promoting stability by enhancing translational read-through, MoMLV RNase $\mathrm{H}$ may have a more direct role in protecting viral mRNAs from decay. Upf1 interacts with the C-terminal domain of eRF1 and the GTPase domain of eRF3 to trigger $\mathrm{NMD}^{27,28}$. Our structure shows that MoMLV RT interacts with eRF1 to occlude eRF3 and suppress termination. Therefore, the MoMLV RT-eRF1 interaction may prevent the recruitment of Upf1 to the terminating ribosome by disrupting the Upf1-eRF1 interaction, thereby allowing the gag-pol mRNA to bypass degradation by NMD.

The RNase $\mathrm{H}$ domain is structurally well conserved among retroviral RTs and exhibits similar substrate recognition activity (Fig. 6a,b). Sequence alignment of helix $\alpha 2$ in RNase $H$ domains 
a

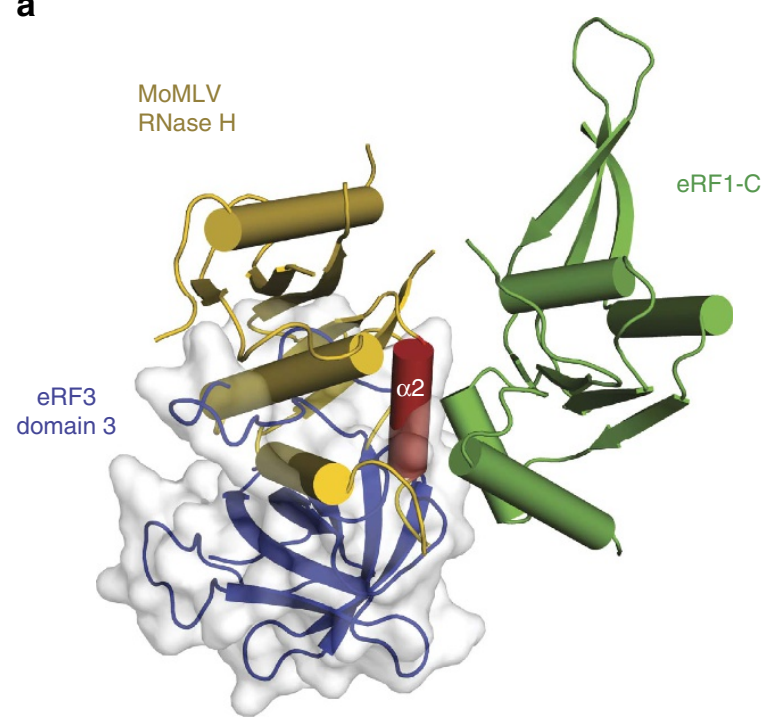

b

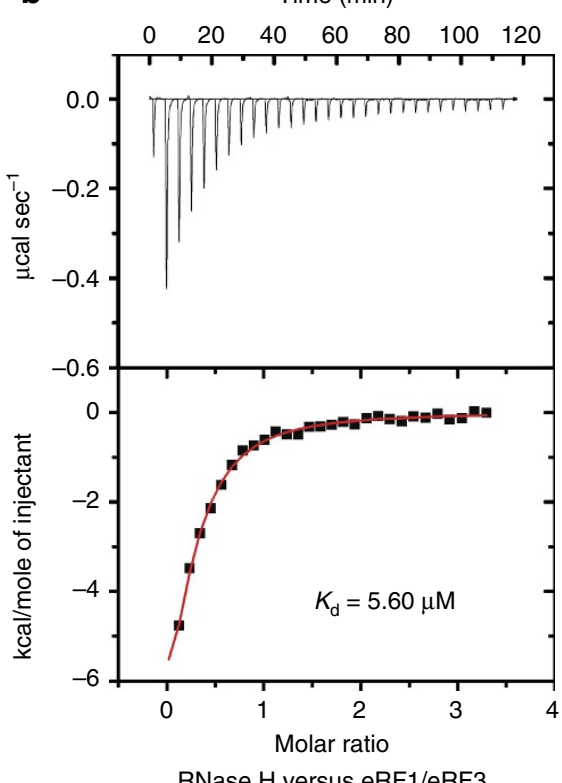

C

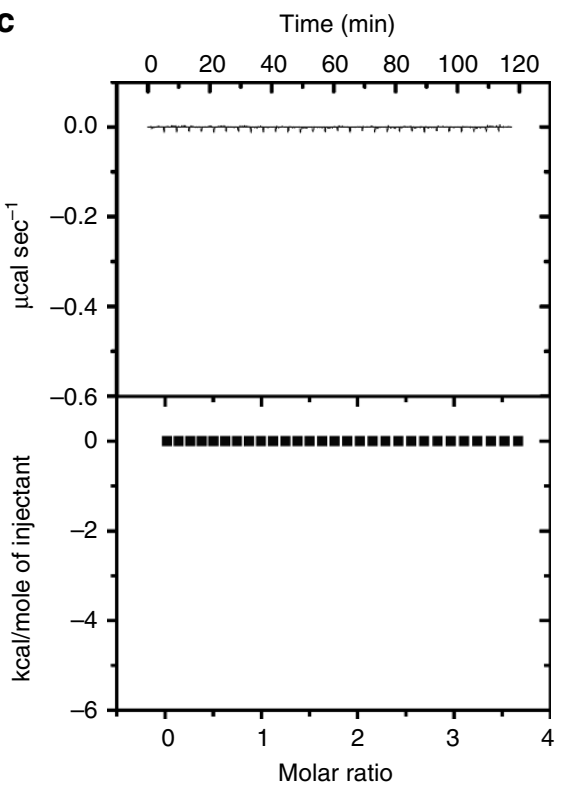

RNase H A589K versus eRF1/eRF3

Figure 4 | The RNase $\mathbf{H}$ domain of MoMLV RT outcompetes eRF3 for binding to eRF1. (a) Superposition of the RNase H/eRF1-C complex with the eRF1/eRF3 complex (PDB accession code: 3E1Y) at eRF1-C domain. The overlapping interface suggests that MoMLV RT and eRF3 are mutually exclusive for binding to eRF1. eRF3 domain 3 is shown in cartoon (blue) covered with grey transparent surface. eRF3 domain 2 and eRF1-C domain in eRF1/eRF3 complex are not displayed for clarity. (b,c) Representative ITC titrations of WT RNase H and mutant A589K to the eRF1/eRF3 complex. The upper panels show the binding isotherms and the lower panels show the integrated heat for each injection fitted to a single-site model.

from various retroviruses reveals that the three eRF1-interacting amino acids of MoMLV RT-R585, F588 and A589-are identical in gammaretroviruses, which utilize read-through for Gag-Pol production but are highly variable in other genera of retroviruses that utilize frameshifting (Fig. 6c; Supplementary Fig. 3a). The residues equivalent to both R585 and F588 are replaced by an alanine in alpharetrovirus RSV and betaretrovirus Mason pfizer monkey virus (MPMV), while the residues equivalent to A589 are substituted by a lysine in RSV, a histidine in MPMV and a glutamate in spumaretrovirus human foamy virus. This observation and our ITC data described above suggest that R585, F588 and A589 in MoMLV RT are structural determinants for its interaction with eRF1, and alterations of these key residues in other genera of retroviruses would render their RTs being unable to bind to eRF1.
The conformational flexibility of the retroviral RNase $\mathrm{H}$ domain, which represents the major difference between the monomeric and dimeric retroviral $\mathrm{RTs}^{10}$, may also account for the species-dependent specificity of the MoMLV RT-eRF1 interaction. The RNase $\mathrm{H}$ domain in monomeric RTs is highly flexible and disordered in previously solved monomeric RT structures including MoMLV RT in complex with dsDNA ${ }^{20}$ and XMRV RT in complex with a DNA/RNA hybrid ${ }^{10}$. Our MoMLV RT/eRF1 structure reveals the full-length monomeric RT architecture for the first time, made possible by the fact that the orientation of the RNase $\mathrm{H}$ domain is fixed upon binding eRF1. Structural superposition of MoMLV and HIV RTs based on the polymerase domain showed that MoMLV RNase $H$ swings $92^{\circ}$ to a completely different position, accompanied by a moderate movement of thumb and connection subdomains 
a
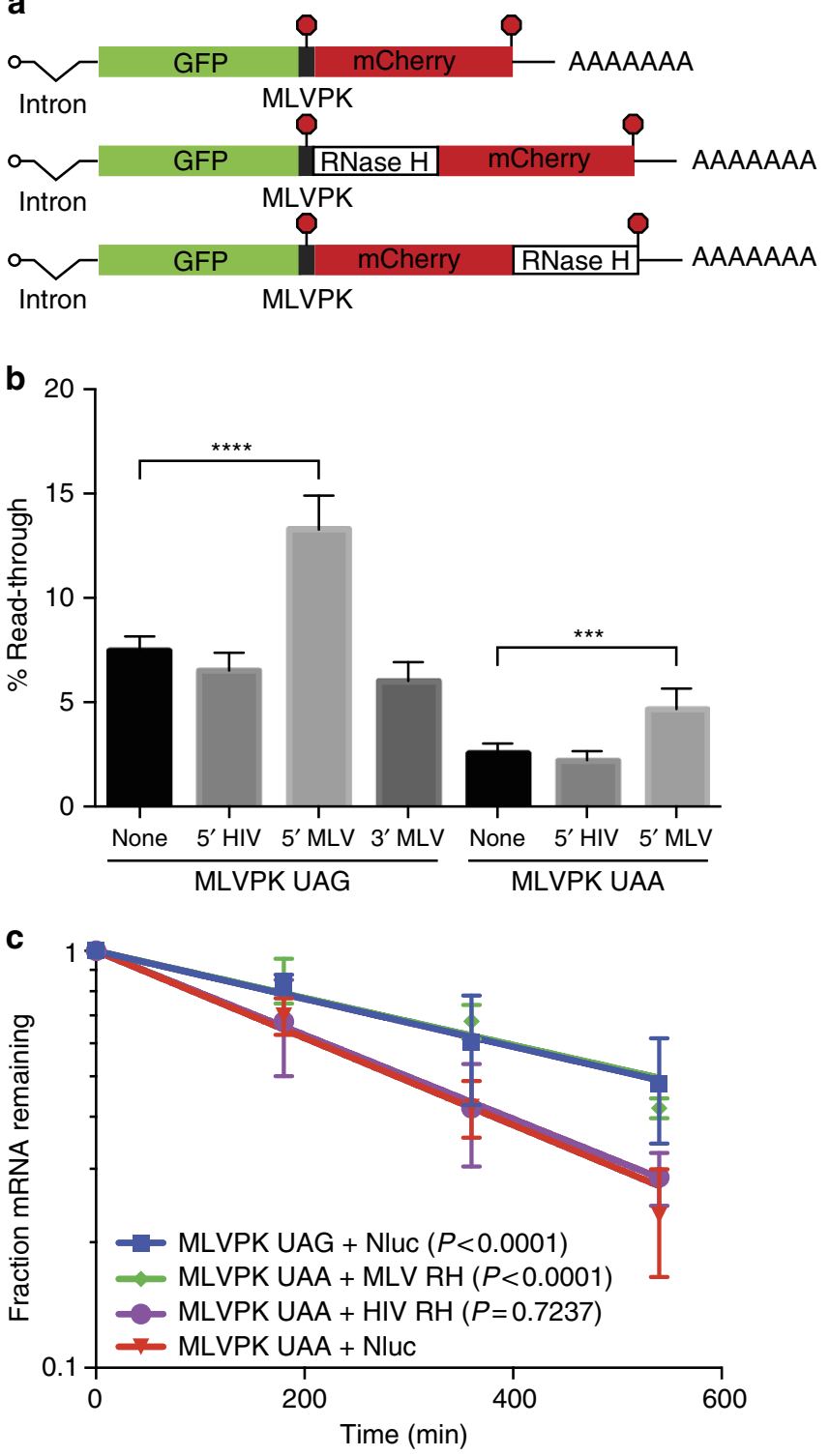

Figure 5 | MoMLV RNase $\mathbf{H}$ enhances stop codon read-through and stabilizes mRNAs. (a) Schematic of tet-regulated reporter mRNAs used in read-through and mRNA decay assays. The indicated $\mathrm{RNase} H$ variants were inserted downstream of the MLVPK, in-frame with the GFP and mCherry ORFs. The red dot represents the stop codon. (b) Translational read-through assays using dual-fluorescent-protein reporters containing the indicated MLVPK and RNase $\mathrm{H}$ variants. The ratio of mCherry:GFP from each experimental construct was normalized to that arising from a sequence-matched control lacking a stop codon between GFP and mCherry. Error bars indicate s.d. $\left(n=7 ;{ }^{\star \star \star \star} P<0.0001 ;{ }^{\star \star \star} P<0.001\right.$ in one-way ANOVA with Sidak's multiple comparisons test). (c) Semi-log plot of levels of the indicated tet-regulated mRNAs throughout pulse-chase mRNA decay assays. Best-fit lines calculated by the least-squares method are indicated; error bars denote s.d. ( $n=4 ; P$ values were calculated using ANCOVA analysis, comparing the indicated mRNAs to MLVPK UAA + Nluc).

(Fig. 6d). This movement is conferred by a 32 amino acid linker region between the connection and RNase $\mathrm{H}$ domains of MoMLV $\mathrm{RT}$, whereas this loop region is not found in HIV RT and is relatively short in other genera of retroviruses (Supplementary Fig. 3b). Moreover, the RNase $\mathrm{H}$ domain of HIV RT is spatially locked by the p51 subunit, and consequently its helix $\alpha 2$ is not accessible for binding eRF1 (Fig. 6d; Supplementary Fig. 3c).
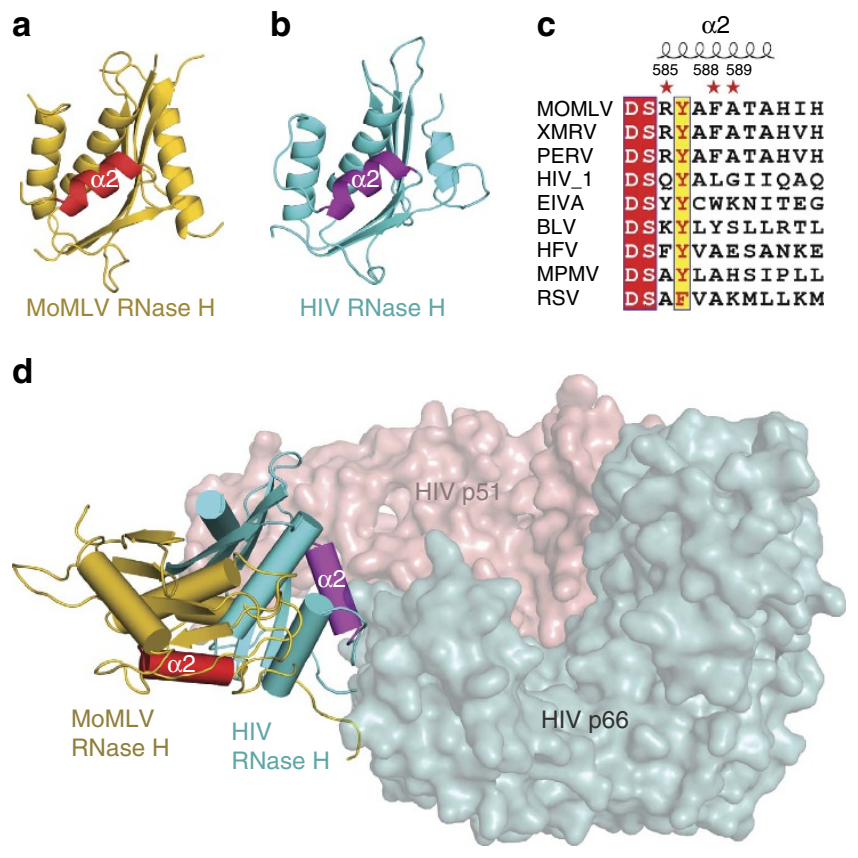

Figure 6 | Structural explanation of why HIV RT cannot interact with eRF1. (a) Cartoon representation of the RNase H domain of MoMLV RT. (b) The RNase H domain of HIV RT, which is coloured in cyan with its helix $\alpha 2$ highlighted in purple. (c) Sequence alignment of the helix $\alpha 2$ from various genera of retroviruses. MoMLV, XMRV and PERV (Porcine endogenous retrovirus) belong to gammaretrovirus. HIV-1 and EIVA (Equine infectious anaemia) are lentivirus. BLV (Bovine leukemia virus), HFV (Human foamy virus), MPMV (Mason pfizer monkey virus) and RSV (Rous sarcoma virus) belong to deltaretrovirus, spumaretrovirus, betaretrovirus and alpharetrovirus respectively. (d) Superposition of MoMLV RT and HIV RT p66 (PDB accession code: 1HYS) at their polymerase domains. HIV RT p66 except RNase $H$ domain and p51 subunits are shown as surfaces coloured in cyan and salmon, respectively.

The presence of a long flexible linker that is essential for MoMLV viral replication $^{29}$, together with the lack of a p51-like subunit to restrict $\mathrm{RNase} \mathrm{H}$ movement, accounts for the wide-range of RNase $\mathrm{H}$ motion observed in MoMLV RT. Taken together, these data suggest that translation termination suppression mediated by the RT-eRF1 interaction appears to represent a unique tactic used by gammaretroviruses, typified by MoMLV.

The expression levels of Gag and Gag-Pol in most retroviruses are delicately balanced to maintain at a ratio close to $20: 1$, which is critical for infection ${ }^{2}$. The production of Gag-Pol results from a translational recoding event, which involves either ribosomal frameshifting or read-through of the gag stop codon ${ }^{30}$. In MoMLV, although a RNA pseudoknot structure downstream of the gag stop codon directs the read-through process ${ }^{6,31}$, the maximal efficiency of read-through also requires the MoMLV RT/eRF1 interaction ${ }^{19}$. The binding of eRF1 by MoMLV RT upregulates read-through, thereby creating a positive feedback loop to drive the synthesis of more Gag-Pol. The effect in the context of the full-length viral mRNA may be strongest in cis, with the nascent Gag-Pol precursor reaching back to interact with eRF1, thereby enhancing read-through for the next ribosome coming along (Supplementary Fig. 4). The outcompetition of eRF3 by MoMLV RT for eRF1 binding would be expected to require a high local concentration of MoMLV RT, as the binding affinity of MoMLV RT to eRF1 is much weaker than that of eRF3 to eRF1. The ultimate determinants of the frequency of read-through will lie in the binding equilibriums for all the 
interactions involving the binding of the RNA pseudoknot, RT, eRF1/eRF3, mRNA as well as ABCE1 to the terminating ribosome in such a feedback loop.

\section{Methods}

Protein expression and purification. Full-length mouse eRF1 (sharing 100\% sequence identity with human eRF1) and its N-, M- and C-domains, as well as MoMLV RT lacking its N-terminal region (aa 24-671), an isolated RT polymerase domain (aa 24-499) and an isolated RT RNase H domain (aa 500-671) were cloned into the vector pGEX-6P-1 (GE Healthcare) and expressed as GST fusion proteins in E. coli BL21-CodonPlus(DE3)-RIL strain (Agilent Technologies). The mutants were created using QuikChange II XL Site-Directed Mutagenesis Kit (Agilent Technologies). WT and mutant proteins were purified by Glutathione Sepharose $4 \mathrm{~B}$ resin, followed by PreScission protease cleavage, ion exchange and gel filtration chromatography (GE Healthcare). To make the RT/eRF1 complex, equal molarity of RT and eRF1 were mixed in gel filtration buffer (20 mM HEPES pH 7.5, $150 \mathrm{mM} \mathrm{NaCl}$ and $2 \mathrm{mM}$ DTT) and loaded into the Hiload Superdex 200 column (GE Healthcare). The fractions containing the complex were pooled and concentrated to around $10 \mathrm{mg} \mathrm{ml}^{-1}$. The RNase H/eRF1-C complex was similarly prepared but purified instead using the Hiload superdex 75 column (GE healthcare) equilibrated with gel filtration buffer (20 mM HEPES pH 7.5, $150 \mathrm{mM} \mathrm{NaCl}$ and $5 \mathrm{mM} \mathrm{DTT}$ ) and concentrated to $65 \mathrm{mg} \mathrm{ml}^{-1}$.

Crystallization and structure determination. The MoMLV RT/eRF1 complex crystals were obtained in the crystallization condition containing $0.02 \mathrm{M}$ magnesium chloride, $0.1 \mathrm{M}$ HEPES $\mathrm{pH} 7.5,20 \%$ polyacrylic acid sodium salt 5100 by the hanging-drop vapour diffusion method at $4^{\circ} \mathrm{C}$. The crystals were cyroprotected by the mother liquor supplemented with $25 \%(\mathrm{v} / \mathrm{v})$ ethylene glycol before flash freezing in liquid nitrogen. The RNase H/eRF1-C complex crystals were grown by mixing $1 \mu \mathrm{l}$ protein with $1 \mu \mathrm{l}$ reservoir solution containing $0.1 \mathrm{M}$ Tris $\mathrm{pH} 8.5$ and $18 \%$ ammonium dihydrogen phosphate at $15^{\circ} \mathrm{C}$ and $25 \%(\mathrm{v} / \mathrm{v})$ glycerol supplemented reservoir condition was used as cryo-protectant. The X-ray diffraction data of the MoMLV RT/eRF1 complex crystals were collected on the beamline ID-23-1 (ESRF, Grenoble, France) and beamline PX-I (SLS, PSI, Switzerland). The diffraction images of the RNase H/eRF1-C complex crystals were collected on beamline I02 (DLS, UK and the beamline BL13B1 (NSRRC, Taiwan)).
The data were processed by the XDS programme ${ }^{32}$. Both structures were solved by molecular replacement with PHASER ${ }^{33}$. The models were further manually built using COOT ${ }^{34}$, refined by $\mathrm{CNS}^{35}$ using the DEN methodology ${ }^{36}$, Phenix ${ }^{37}$ and REFMAC5 (ref. 38). Structure validation was performed using PROCHECK ${ }^{39}$. The statistics for the diffraction data and structure refinement are listed in Table 1. Stereo image of a representative part of electron density map around the RNase $\mathrm{H}$ helix $\alpha 2$ is shown in Supplementary Fig. 5 .

In vitro binding experiments. Overall, $50 \mu \mathrm{l}$ beads bound with WT GST-tagged MoMLV RNase $\mathrm{H}$ and its mutants were incubated with $1 \mu \mathrm{g}$ of purified His-tagged eRF1-C protein at $4^{\circ} \mathrm{C}$ for $1 \mathrm{~h}$ in buffer containing $20 \mathrm{mM}$ HEPES $\mathrm{pH} 7.5$ and $150 \mathrm{mM} \mathrm{NaCl}$. The beads were washed several times and the bound proteins were analyzed by SDS-PAGE gel and western blotting.

Isothermal titration calorimetry. ITC measurements were performed at $22{ }^{\circ} \mathrm{C}$ using MicroCal VP-ITC (MicroCal Inc.). Protein samples were dialyzed into a buffer containing $20 \mathrm{mM}$ HEPES pH 7.5, $150 \mathrm{mM} \mathrm{NaCl}$ and $5 \mathrm{mM}$ TCEP. A sample syringe with stirring speed of 290 r.p.m. was used to titrate the injectant protein (220-300 $\mu \mathrm{M}$ ) into a cell containing $13-20 \mu \mathrm{M}$ protein. The titration comprised 29 injections of $10 \mu \mathrm{l}$ each, separated by $240 \mathrm{~s}$ equilibration time. The datasets were analyzed using the Origin 7.0 program, fitted to a single-site binding model.

RNase $\mathbf{H}$ activity assays. RNase $\mathrm{H}$ activity assays were carried out as previously described ${ }^{40}$. A RNA template ( $5^{\prime}$ UCUUUUCAACGACGAAAAGA $3^{\prime}$ ) was heat annealed to a threefold molar excess of DNA primer $\left(5^{\prime}\right.$ TCTTTTCGTTG $\left.3^{\prime}\right)$ to yield a RNA-DNA duplex substrate. MoMLV RNase $\mathrm{H}$ was incubated with RNA-DNA duplex at $37^{\circ} \mathrm{C}$ in buffer containing $50 \mathrm{mM}$ Tris (pH 8.0), $60 \mathrm{mM} \mathrm{KCl}$, $0.1 \mathrm{mg} \mathrm{ml}^{-1}$ bovine serum albumin, $2 \mathrm{mM}$ dithiothreitol and $0.5 \mathrm{mM}$ EDTA. Reactions were initiated by the addition of $0.5 \mathrm{mM} \mathrm{MnCl}_{2}$ and were stopped at 10 min by adding an equal volume of $100 \%$ formamide containing bromophenol blue. RNase $\mathrm{H}$ cleavage products were resolved on $15 \%$ polyacrylamide $-7 \mathrm{M}$ urea gels and stained by GelRed (Biotium).

Virus purification and virus replication assays. WT pNCA was described previously ${ }^{19}$. Lenti-X 293 T (Clontech) and Rat2 cells were maintained in DMEM

Table 1 | Diffraction data collection and refinement statistics.

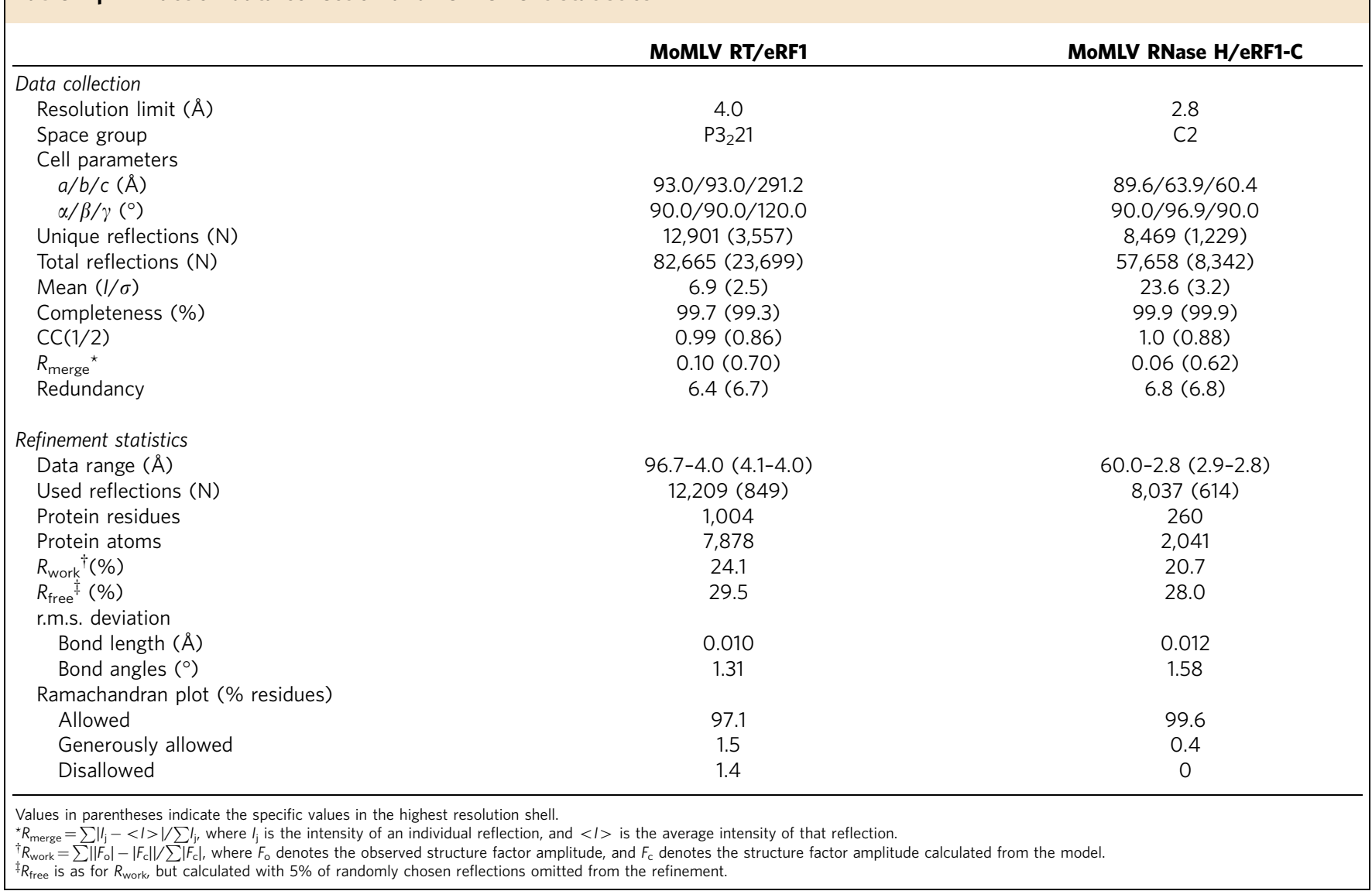


medium supplemented with 10\% FBS. For virus packaging, Lenti-X 293 T cells in $60 \mathrm{~mm}$ dishes were transfected with $5 \mu \mathrm{g}$ plasmids using Lipofectamine LTX with Plus Reagent (Life Technologies). Two days after transfection, cells were lysed for western blot to analyze the expression of MoMLV proteins. The supernatant of transfected cells was filtered through $0.45 \mathrm{~mm}$ membrane and further ultra-centrifuged through the $25 \%$ sucrose cushion at 35,000 r.p.m. for $2 \mathrm{~h}$ to purify the virus. For replication assay, Rat2 cells in 6-well plate were infected with $100 \mathrm{ml}$ supernatants from MoMLV provirus DNA (WT or mutants) transfected $293 \mathrm{~T}$ cells for $3 \mathrm{~h}$. The supernatants were taken out everyday after infection to detect the reverse transcriptase activities. pNCS-3Myc-Pro ${ }^{\text {D27S }}$ was constructed from pNCS-3Myc by mutating the aspartate in the protease catalytic site to serine to eliminate the protease activity. pNCS-3Myc was provided by Dr Eran Bacharach (Tel Aviv University, Tel Aviv, Israel), which contains three myc tag repeats in the p12 domain of Gag as described previously ${ }^{41}$.

Read-through and mRNA decay assays. To create vectors for read-through and mRNA decay assays, the EGFP and mCherry were cloned into the tetracyclineregulated pcTET2 $\beta \mathrm{wt} \beta$ vector $^{42}$, replacing all $\beta$-globin sequence. The hybrid intron from the $\mathrm{pCI}$ vector (Promega) was amplified by PCR and inserted into the HindIII site of the resulting vector to create pcTET2iFP. The indicated MLVPK variants were inserted between the GFP and mCherry ORFs, placing the stop codon in-frame. Control constructs in which the stop codon was replaced by a CAG codon were generated for each sequence variant. Codon-optimized RNase $\mathrm{H}$ domains from HIV-1 or MoMLV containing the catalytic D524N mutation or Nano luciferase (Promega) were cloned in-frame downstream of MLVPK or mCherry as indicated.

For dual-fluorescent-protein read-through assays, 293 Tet-off cells (Clontech) were maintained in DMEM supplemented with $10 \%$ FBS, and transfected with Turbofect (ThermoFisher) according to the manufacturer's instructions. Cells were collected $48 \mathrm{~h}$ post transfection in $1 \times$ passive lysis buffer (Promega), and fluorescence was measured on a Tecan Infinite F200 plate reader. Read-through efficiency was determined by normalizing the ratio of GFP:mCherry fluorescence from the experimental construct to GFP:mCherry fluorescence from a sequencematched control plasmid lacking a termination codon between GFP and mCherry, as previously described ${ }^{43}$.

mRNA decay assays were performed using Hela Tet-off cells (Clontech) as previously described ${ }^{22}$ with minor modifications. Cells were split to $60 \mathrm{mM}$ plates at a density of $5 \times 10^{5}$ cells per plate and Turbofect (ThermoFisher) was used for transfection with $800 \mathrm{ng}$ of the indicated experimental vector, $200 \mathrm{ng}$ of pcDNA GFP TP ${ }^{22}$ as a co-transfection control, and $500 \mathrm{ng}$ pcDNA 3.1 empty vector, in the presence of $2 \mathrm{ng} \mathrm{ml}^{-1}$ doxycycline. Following a 24-h incubation, cells were transferred to 12 -well plates. The next day, cells were washed into DMEM $+10 \%$ tetracycline-free FBS (Clontech) for $4 \mathrm{~h}$. Transcription was halted with $1 \mu \mathrm{g} \mathrm{ml}^{-1}$ doxycycline, and timepoints were collected in Trizol (Life Technologies) after $30 \mathrm{~min}$ and at $3 \mathrm{~h}$ intervals thereafter. Northern blotting was performed as described using hexamer-labelled DNA probes against the full GFP sequence ${ }^{22}$, detected using a Storm 865 phosphorimager (GE Healthcare), and quantified on ImageStudio software (Li-Cor). Half-lives were determined from the best-fit line to semi-log plots of relative RNA abundances over time, and statistical significance was calculated by the ANCOVA analysis using Prism software (Graphpad).

Data availability. The atomic coordinates and structural factors for MoMLV RT/eRF1 and MoMLV RNase H/eRF1-C have been deposited with the Protein Data Bank under accession codes 5DMQ and 5DMR, respectively. All other data are available from the corresponding authors on reasonable request.

\section{References}

1. Walsh, D. \& Mohr, I. Viral subversion of the host protein synthesis machinery. Nat. Rev. Microbiol. 9, 860-875 (2011).

2. Shehu-Xhilaga, M., Crowe, S. M. \& Mak, J. Maintenance of the Gag/Gag-Pol ratio is important for human immunodeficiency virus type 1 RNA dimerization and viral infectivity. J. Virol. 75, 1834-1841 (2001).

3. Felsenstein, K. M. \& Goff, S. P. Expression of the gag-pol fusion protein of Moloney murine leukemia virus without gag protein does not induce virion formation or proteolytic processing. J. Virol. 62, 2179-2182 (1988).

4. Feng, Y. X., Yuan, H., Rein, A. \& Levin, J. G. Bipartite signal for read-through suppression in murine leukemia virus mRNA: an eight-nucleotide purine-rich sequence immediately downstream of the gag termination codon followed by an RNA pseudoknot. J. Virol. 66, 5127-5132 (1992).

5. Felsenstein, K. M. \& Goff, S. P. Mutational analysis of the gag-pol junction of Moloney murine leukemia virus: requirements for expression of the gag-pol fusion protein. J. Virol. 66, 6601-6608 (1992).

6. Wills, N. M., Gesteland, R. F. \& Atkins, J. F. Evidence that a downstream pseudoknot is required for translational read-through of the Moloney murine leukemia virus gag stop codon. Proc. Natl Acad. Sci. USA 88, 6991-6995 (1991).

7. ten Dam, E. B., Pleij, C. W. \& Bosch, L. RNA pseudoknots: translational frameshifting and readthrough on viral RNAs. Virus Genes 4, 121-136 (1990).

8. Houck-Loomis, B. et al. An equilibrium-dependent retroviral mRNA switch regulates translational recoding. Nature 480, 561-564 (2011).
9. Goff, S. P. Retroviral reverse transcriptase: synthesis, structure, and function. J. Acquir. Immune Defic. Syndr. 3, 817-831 (1990).

10. Nowak, E. et al. Structural analysis of monomeric retroviral reverse transcriptase in complex with an RNA/DNA hybrid. Nucleic Acids Res. 41, 3874-3887 (2013).

11. Huang, H., Chopra, R., Verdine, G. L. \& Harrison, S. C. Structure of a covalently trapped catalytic complex of HIV-1 reverse transcriptase: implications for drug resistance. Science 282, 1669-1675 (1998).

12. Dever, T. E. \& Green, R. The elongation, termination, and recycling phases of translation in eukaryotes. Cold Spring Harb. Perspect. Biol. 4, a013706 (2012).

13. Preis, A. et al. Cryoelectron microscopic structures of eukaryotic translation termination complexes containing eRF1-eRF3 or eRF1-ABCE1. Cell Rep. 8, 59-65 (2014).

14. des Georges, A. et al. Structure of the mammalian ribosomal pre-termination complex associated with eRF1.eRF3.GDPNP. Nucleic Acids Res. 42, 3409-3418 (2014).

15. Taylor, D. et al. Cryo-EM structure of the mammalian eukaryotic release factor eRF1-eRF3-associated termination complex. Proc. Natl Acad. Sci. USA 109, 18413-18418 (2012).

16. Cheng, Z. et al. Structural insights into eRF3 and stop codon recognition by eRF1. Genes Dev. 23, 1106-1118 (2009).

17. Song, H. et al. The crystal structure of human eukaryotic release factor eRF1-mechanism of stop codon recognition and peptidyl-tRNA hydrolysis. Cell 100, 311-321 (2000).

18. Kong, C. et al. Crystal structure and functional analysis of the eukaryotic class II release factor eRF3 from S. pombe. Mol. Cell 14, 233-245 (2004).

19. Orlova, M., Yueh, A., Leung, J. \& Goff, S. P. Reverse transcriptase of Moloney murine leukemia virus binds to eukaryotic release factor 1 to modulate suppression of translational termination. Cell 115, 319-331 (2003).

20. Das, D. \& Georgiadis, M. M. The crystal structure of the monomeric reverse transcriptase from Moloney murine leukemia virus. Structure 12, 819-829 (2004).

21. Smith, J. E. \& Baker, K. E. Nonsense-mediated RNA decay--a switch and dial for regulating gene expression. BioEssays 37, 612-623 (2015).

22. Hogg, J. R. \& Goff, S. P. Upf1 senses $3^{\prime}$ UTR length to potentiate mRNA decay. Cell 143, 379-389 (2010).

23. Pisarev, A. V. et al. The role of ABCE1 in eukaryotic posttermination ribosomal recycling. Mol. Cell 37, 196-210 (2010).

24. Shoemaker, C. J. \& Green, R. Kinetic analysis reveals the ordered coupling of translation termination and ribosome recycling in yeast. Proc. Natl Acad. Sci. USA 108, E1392-E1398 (2011).

25. Ge, Z., Quek, B. L., Beemon, K. L. \& Hogg, J. R. Polypyrimidine tract binding protein 1 protects mRNAs from recognition by the nonsense-mediated mRNA decay pathway. eLife 5, e11155 (2016).

26. Mocquet, V. et al. The human T-lymphotropic virus type 1 tax protein inhibits nonsense-mediated mRNA decay by interacting with INT6/EIF3E and UPF1. J. Virol. 86, 7530-7543 (2012).

27. Ivanov, P. V., Gehring, N. H., Kunz, J. B., Hentze, M. W. \& Kulozik, A. E. Interactions between UPF1, eRFs, PABP and the exon junction complex sugges an integrated model for mammalian NMD pathways. EMBO J. 27, 736-747 (2008).

28. Czaplinski, K. et al. The surveillance complex interacts with the translation release factors to enhance termination and degrade aberrant mRNAs. Genes Dev. 12, 1665-1677 (1998)

29. Puglia, J. et al. Revealing domain structure through linker-scanning analysis of the murine leukemia virus (MuLV) RNase $\mathrm{H}$ and MuLV and human immunodeficiency virus type 1 integrase proteins. J. Virol. 80, 9497-9510 (2006).

30. Baranov, P. V., Gesteland, R. F. \& Atkins, J. F. Recoding: translational bifurcations in gene expression. Gene 286, 187-201 (2002).

31. Wills, N. M., Gesteland, R. F. \& Atkins, J. F. Evidence that a downstream pseudoknot is required for translational read-through of the Moloney murine leukemia virus gag stop codon. Proc. Natl Acad. Sci. U S A 88, 6991-6995 (1991).

32. Kabsch, W. Xds. Acta. Crystallogr. D Biol. Crystallogr. 66, 125-132 (2010).

33. Storoni, L. C., McCoy, A. J. \& Read, R. J. Likelihood-enhanced fast rotation functions. Acta. Crystallogr. D Biol. Crystallogr. 60, 432-438 (2004).

34. Emsley, P. \& Cowtan, K. Coot: model-building tools for molecular graphics. Acta. Crystallogr. D Biol. Crystallogr. 60, 2126-2132 (2004).

35. Brunger, A. T. et al. Crystallography \& NMR system: a new software suite for macromolecular structure determination. Acta. Crystallogr. D Biol. Crystallogr. 54, 905-921 (1998).

36. Schroder, G. F., Levitt, M. \& Brunger, A. T. Super-resolution biomolecular crystallography with low-resolution data. Nature 464, 1218-1222 (2010).

37. Adams, P. D. et al. PHENIX: a comprehensive Python-based system for macromolecular structure solution. Acta. Crystallogr. D Biol. Crystallogr. 66, 213-221 (2010). 
38. Murshudov, G. N. et al. REFMAC5 for the refinement of macromolecular crystal structures. Acta. Crystallogr. D Biol. Crystallogr. 67, 355-367 (2011).

39. Laskowski, R. A., Macarthur, M. W., Moss, D. S. \& JM, T. PROCHECK: A program to check the stereochemical quality of protein structures. J. Appl. Cryst. 26, 283-291 (1993).

40. Kirby, K. A. et al. Structural and inhibition studies of the RNase H function of xenotropic murine leukemia virus-related virus reverse transcriptase. Antimicrob. Agents Chemother. 56, 2048-2061 (2012).

41. Prizan-Ravid, A. et al. The Gag cleavage product, p12, is a functional constituent of the murine leukemia virus pre-integration complex. PLoS Pathog. 6, e1001183 (2010).

42. Lykke-Andersen, J., Shu, M. D. \& Steitz, J. A. Communication of the position of exon-exon junctions to the mRNA surveillance machinery by the protein RNPS1. Science 293, 1836-1839 (2001).

43. Grentzmann, G., Ingram, J. A., Kelly, P. J., Gesteland, R. F. \& Atkins, J. F. A dual-luciferase reporter system for studying recoding signals. RNA 4, 479-486 (1998).

\section{Acknowledgements}

We thank the beamline scientists at ID-23-1 (ESRF, Grenoble, France), beamline PX-I (SLS, PSI, Switzerland), BL13B1 (NSRRC, Taiwan) and beamline I02 (DLS, UK) for assistance and access to synchrotron radiation facilities. We thank Dr Stephen Hughes for generously providing plasmid of HIV RT. This work was supported by the Agency for Science, Technology and Research in Singapore (H.S.), in part by the Intramural Research Program of the NIH, NHLBI (J.R.H.) and by the NCI Grant R01 CA 30488 from the National Cancer Institute (S.P.G.). S.P.G. is an investigator of the Howard Hughes Medical Institute.

\section{Author contributions}

H.S., S.P.G. and J.R.H. designed the study. X.T., Y.Z., S.L.B., M.W.B., B.C. and C.C. performed the experiments. X.T., Y.Z., S.L.B., J.R.H. and H.S. analyzed the data. X.T., H.S., J.R.H. and S.P.G. wrote the manuscript.

\section{Additional information}

Supplementary Information accompanies this paper at http://www.nature.com/ naturecommunications

Competing financial interests: The authors declare no competing financial interests.

Reprints and permission information is available online at http://npg.nature.com/ reprintsandpermissions/

How to cite this article: Tang, X. et al. Structural basis of suppression of host translation termination by Moloney Murine Leukemia Virus. Nat. Commun. 7:12070 doi: $10.1038 /$ ncomms12070 (2016).

(c) (i) International License. The images or other third party material in this article are included in the article's Creative Commons license, unless indicated otherwise in the credit line; if the material is not included under the Creative Commons license, users will need to obtain permission from the license holder to reproduce the material. To view a copy of this license, visit http://creativecommons.org/licenses/by/4.0/

(C) The Author(s) 2016 